\title{
Cubic velocity feedback control of high-amplitude vibration of a nonlinear plant to a primary resonance excitation
}

\author{
Li Jun*, Shen Rongying and Hua Hongxing \\ Vibration, Shock \& Noise Institute, Shanghai Jiao Tong University, 800 Dongchuan Road, Shanghai 200240, P.R. \\ China
}

Received 16 May 2005

Revised 10 December 2005

\begin{abstract}
High-amplitude response suppression of the primary resonance of a nonlinear plant under cubic velocity feedback control is investigated. By means of the multiple scales method, two equations on the amplitude and phase of the response of the nonlinear system are obtained and the force-response and frequency-response curves are shown. The stability analyses for the open- and closed-loop responses of the system are carried out and the performance of the control strategy is investigated. The instantaneous power requirement of the control law is also examined. It can be demonstrated that appropriate choice for the feedback gain can greatly reduce the response amplitude of the primary resonance and completely eliminate the multiple responses. Finally the perturbation solutions are verified with numerical simulations.
\end{abstract}

Keywords: Active control, nonlinear velocity feedback, perturbation method, primary resonance

\section{Introduction}

With the advances in design methods and structural materials, modern systems need to face higher disturbances with lower damping and increased compliance, resulting in larger and often nonlinear vibrations. Thus, modeling of the dynamic behavior of the vibrating systems must be generalized to include nonlinear effects. In fact, nonlinear effects exist in almost all vibration systems and sometimes cannot be ignored, especially for the cases in which the level of excitation results in deflections beyond the linear region. In contrast to the linear theory, the nonlinear behavior of a vibrating system depends on initial conditions. Analytical and experimental results have indicated that the nonlinear systems, when subjected to dynamic loads, may display a wealth of phenomena including subharmonic and superharmonic oscillations, period multiplying bifurcations, co-existing small and large amplitude oscillations in resonant regions, limit cycles, chaotic vibrations, and dynamic jumps [1,2]. These typical nonlinear behaviors may cause unacceptable vibrations and are often dangerous and undesirable. The high-amplitude vibrations often lead to material fatigue, structural damage and possibly affecting structural safety, deterioration of system performance, and increased noise level. These effects are usually prominent around the natural frequencies of the system. External resonant excitation is one of the sources of undesirable vibrations.

The high-amplitude nonlinear vibrations of the systems usually cannot be tolerated and should be avoided whenever possible. To achieve this goal, developing a strategy for reducing these vibrations has been a major focus of research. The advances in modern control techniques have led to the application of active control strategies as an interesting option in protecting the systems from undesirable nonlinear vibrations. In its simplest form, active control consists

${ }^{*}$ Corresponding author. E-mail: LYJ60023@yahoo.com. 
of sensors, electronic circuits, and actuators. The sensor feeds the electronic circuit with a feedback signal which is proportional to the vibration level of the plant. The output of the electronic circuit is the actuation force law, which is fed into the actuator. The actuator, in turn, applies the opposing force on the plant.

Over the last few decades, remarkable progress has been made in active structural control theory [3]. Numerous papers have been dedicated to the control of resonantly forced systems in various engineering fields. However, most of these studies are mainly concerned with linear control of linear problems. The analysis of the vibration control of eminently nonlinear structures is relatively rarely attempted in the literature. This is mainly due to the inherent complexity of this class of problems. Furthermore, due to the complexity of real-life structures, it is not always easy and inexpensive to build a prototype for these structures. Hence, theoretically and numerically simulating the controlled behavior of such structures become extremely important before actually implementing the active control strategy. Some interesting strategies have been proposed lately for the active nonlinear control of engineering systems [4-7]. Moiola et al. [8] considered the Hopf bifurcations in the nonlinear feedback systems with time delay. $\mathrm{Hu}$ et al. [9] considered the primary resonance and the 1/3 subharmonic resonance of a forced Duffing oscillator with time delay state feedback. El-Badawy and Nayfeh [10] used two control laws based on linear velocity feedback and cubic velocity feedback to suppress the high-amplitude vibrations of a structural dynamic model of the twin-tail assembly of an F-15 fighter when subjected to primary resonance excitations. Pinto and Goncalves [11] investigated the active control of the non-linear vibration of a simply supported buckled beam under lateral loading. To calculate the control moments which were applied along the beam axis, a control strategy based on a nonlinear optimal control theory using state feedback with an indicial formulation was implemented. Maccari $[12,13]$ investigated the response of a parametrically or externally excited van der Pol oscillator and it was shown that vibration control and quasiperiodic motion suppression were possible for appropriate choices of the time delay and feedback gain. Yabuno et al. [14] theoretically and experimentally investigated the parametric resonance. They analyzed the system in linear theory and stabilized the vibration by using a velocity feedback. Oueini and Nayfeh [15] proposed single-mode control of a cantilever beam under principal parametric excitation. Inertial and stiffness based nonlinearities, together with nonlinear air damping, were included in the single-mode equation of motion and the system was parametrically excited. Chen et al. [16] proposed a nonlinear control approach that combines the linear velocity and cubic velocity feedback control strategies for the bifurcation control of a flexible cantilever beam subject to a principal parametric excitation.

The purpose of this paper is to present a feasible methodology that can achieve good control performance for a nonlinear dynamic system subjected to a primary resonance excitation. It is well known that accurate modeling of the response of the nonlinear system is important for reliable prediction and control of that response. Here, a more general nonlinear single-degree-of-freedom system is considered, which includes velocity-dependent damping forces, polynomial and differential-polynomial nonlinearities. This model is capable of describing with a reasonable degree of accuracy the nonlinear behaviors of the systems in many engineering applications. Since the system under consideration is nonlinear, linear control theory cannot predict the response of the system, and linear tools cannot be used to choose the optimal gain. Motivated by the results of El-Badawy and Nayfeh [10], a state variable nonlinear feedback control, namely cubic velocity feedback control, is introduced to suppress the large-amplitude vibration of the response of the nonlinear plant excited by a primary resonant excitation. First, the first-order approximate solution of the nonlinear plant is constructed by means of the multiple scales method and the stability analysis is performed. Then, the frequency-response and force-response curves are shown for the open- and closed-loop systems and the performance of the control law is investigated. Next, the instantaneous power requirement of the control strategy is examined. Finally, the numerical simulations of the nonlinear plant are performed to confirm and validate the perturbation solutions.

\section{Perturbation solution}

A nonlinear control law based on cubic velocity feedback is introduced to suppress the large-amplitude vibration of a thin, uniform cantilever beam subjected to a primary resonance excitation. Such beam is usually long and flexible enough to allow for large but still elastic deformation that enforces the contribution of the nonlinear dynamics of the beam. The unimodal form of the nonlinear governing integro-differential equation of motion describing the 
large-amplitude response of the beam is used to model the dynamics of the nonlinear plant, which can be reduced to a nonlinear ordinary differential equation $[15,16]$ via applying any of the variational methods such as Rayleigh-Ritz or Galerkin's method. The closed-loop response of the nonlinear plant to a primary resonance excitation is modeled by the following nonlinear second-order differential equation

$$
\ddot{u}+\omega^{2} u+2 \varepsilon \mu_{1} \dot{u}+\varepsilon \mu_{2} \dot{u}|\dot{u}|+\varepsilon \alpha_{3} u^{3}+\varepsilon \delta \dot{u}^{2} u+\varepsilon \delta u^{2} \ddot{u}=\varepsilon \eta_{1} f \cos \left(\Omega t+\tau_{e}\right)+T
$$

where superscript dot denotes differentiation with respect to time $t, \omega$ is the linear undamped natural frequency, $\mu_{1}$ is the viscous damping coefficient, $\mu_{2}$ is the quadratic damping coefficient that may model structural or fluid $\mathrm{drag}, \alpha_{3}$ is the curvature nonlinearity coefficient, $\delta$ is the inertia nonlinearity coefficients. The direct excitation is harmonic and its effect is modeled using the parameter $\eta_{1}$. The forcing parameters $f, \Omega$, and $\tau_{e}$ model the forcing amplitude, frequency, and phase, respectively. $T$ is the control force. For the nonlinear cubic velocity feedback control, the velocity measurement of the plant is determined by a strain measurement (appropriately differentiated). The controller is then designed to provide a signal to the actuator proportional to the cube of the measured velocity. The actuator (PZT) is then commanded to provide force to the plant. Let $T=-\varepsilon G \dot{u}^{3}$, where $G$ is the positive constant called the feedback gain.

Here, consider the case of primary resonance (i.e., $\Omega \approx \omega$ ). The scaling parameter $\varepsilon$ represents the level to which the nonlinearity has an effect on the system. The nonlinear governing differential equation of motion Eq. (1) does not admit a closed-form exact solution. More often, one has to resort to approximate approaches to predict characteristics, such as steady-state solution and stability of the nonlinear system. To do this, the method of multiple scales [17] is employed to obtain the approximate solution for the response of the system, given in Eq. (1). To differentiate between slow- and fast-varying effects on the system, the scaling parameter $\varepsilon$ is used and the time $t$ is replaced with independent variables $T_{n}$, such that

$$
T_{n}=\varepsilon^{n} t, n=0,1,2, \cdots
$$

where $T_{0}$ represents a fast time scale and $T_{1}, T_{2}, \ldots$ represent slow time scales. The ordinary-differential Eq. (1) now becomes a partial-differential equation, with $u$ being a function of the new time scales. The first- and secondderivatives operators with respect to $t$ are then given by

$$
\begin{aligned}
\frac{d}{d t} & =\frac{d T_{0}}{d t} \frac{\partial}{\partial T_{0}}+\frac{d T_{1}}{d t} \frac{\partial}{\partial T_{1}}+\cdots=D_{0}+\varepsilon D_{1}+\cdots \\
\frac{d^{2}}{d t^{2}} & =\left(D_{0}+\varepsilon D_{1}+\cdots\right)^{2}=D_{0}^{2}+2 \varepsilon D_{0} D_{1}+\cdots
\end{aligned}
$$

The generalized coordinate $u$ is expanded using the scaling parameter, $\varepsilon$, as

$$
u(t ; \varepsilon)=u_{0}\left(T_{0}, T_{1}\right)+\varepsilon u_{1}\left(T_{0}, T_{1}\right)+\cdots
$$

In this manner, a series solution for $u$ of Eq. (1) based on functions that have scaled levels of influence on the total response of the system is assumed. The primary portion of the response is found in $u_{0}$. The perturbation $u_{1}$ serves as a small adjustment to the response, and so on. Substituting Eqs (3)-(5) into Eq. (1) and considering terms up to order $\varepsilon$ obtains

$$
\begin{aligned}
& \left(D_{0}^{2}+2 \varepsilon D_{0} D_{1}\right)\left(u_{0}+\varepsilon u_{1}\right)+\omega^{2}\left(u_{0}+\varepsilon u_{1}\right) \\
& \quad+2 \varepsilon \mu_{1} D_{0} u_{0}+\varepsilon \mu_{2} D_{0} u_{0}\left|D_{0} u_{0}\right|+\varepsilon \alpha_{3} u_{0}^{3}+\varepsilon \delta u_{0}\left(D_{0} u_{0}\right)^{2}+\varepsilon \delta u_{0}^{2} D_{0}^{2} u_{0} \\
& \quad=\varepsilon \eta_{1} f \cos \left(\Omega T_{0}+\tau_{e}\right)-\varepsilon G\left(D_{0} u_{0}\right)^{3}
\end{aligned}
$$

The partial-differential Eq. (6) can be simplified and separated into coefficients of like powers of $\varepsilon$. Again, considering terms up to order $\varepsilon$ yields

$$
\begin{aligned}
& O\left(\varepsilon^{0}\right): \\
& D_{0}^{2} u_{0}+\omega^{2} u_{0}=0 \\
& O\left(\varepsilon^{1}\right):
\end{aligned}
$$




$$
\begin{aligned}
D_{0}^{2} u_{1}+\omega^{2} u_{1}= & -2 D_{0} D_{1} u_{0}-2 \mu_{1} D_{0} u_{0}-\mu_{2} D_{0} u_{0}\left|D_{0} u_{0}\right| \\
& -\alpha_{3} u_{0}^{3}-\delta u_{0}\left(D_{0} u_{0}\right)^{2}-\delta u_{0}^{2} D_{0}^{2} u_{0} \\
& +\eta_{1} f \cos \left(\Omega T_{0}+\tau_{e}\right)-G\left(D_{0} u_{0}\right)^{3}
\end{aligned}
$$

where $O\left(\varepsilon^{j}\right)(j=0,1)$ denotes the power of $\varepsilon$ under consideration.

The solutions of the system of equations given by Eqs (7) and (8) are obtained by substituting the solution of the lower-order equation in $\varepsilon$ into the higher-order equation in $\varepsilon$. First, the solution for $u_{0}$ is obtained from Eq. (7) and then substituted into Eq. (8). This yields an approximate solution of the system that is accurate to first order. Following this procedure, the solution of Eq. (7) is expressed in the following form

$$
\begin{aligned}
u_{0} & =\frac{1}{2} a\left(T_{1}\right) e^{i\left(\beta\left(T_{1}\right)+\omega T_{0}\right)}+c c \\
& =A\left(T_{1}\right) e^{i \omega T_{0}}+c c
\end{aligned}
$$

where $A=\frac{1}{2} a e^{i \beta}, A, a$ and $\beta$ are functions of $T_{1}$, and $c c$ represents the complex conjugate of the preceding terms. In this sense $a$ and $\beta$ represent the amplitude and phase of the response.

As discussed earlier, the case when the excitation frequency $\Omega$ is near the natural frequency $\omega$ of the plant is considered. This relationship is expected as

$$
\Omega=\omega+\varepsilon \sigma
$$

where $\sigma$ is a frequency detuning parameter, which indicates the small difference between the excitation frequency and the natural frequency of the plant.

Substituting the solution for $u_{0}$ from Eqs (9) into (8) obtains

$$
\begin{aligned}
D_{0}^{2} u_{1}+\omega^{2} u_{1}= & {\left[-2 i \omega A^{\prime}-2 i \omega \mu_{1} A-\mu_{2} g_{1}-3 \alpha_{3} A^{2} \bar{A}-\delta \omega^{2} A^{2} \bar{A}+3 \delta \omega^{2} A^{2} \bar{A}\right.} \\
& \left.+\frac{1}{2} \eta_{1} f e^{i\left(\sigma T_{1}+\tau_{e}\right)}-3 i G \omega^{3} A^{2} \bar{A}\right] e^{i \omega T_{0}}+N S T+c c
\end{aligned}
$$

where NST denotes terms that do not produce secular terms and

$$
g_{1}=-\frac{1}{2 \pi} \int_{0}^{2 \pi}\left(i \omega A e^{i \omega T_{0}}-i \omega \bar{A} e^{-i \omega T_{0}}\right)\left|i \omega A e^{i \omega T_{0}}-i \omega \bar{A} e^{-i \omega T_{0}}\right| e^{-i \omega T_{0}} d\left(\omega T_{0}\right)
$$

To ensure a uniform expansion for $u$, the occurrence of secular terms, or terms that cause series divergence should be prevented. This condition requires setting the coefficients of $e^{ \pm i \omega T_{0}}$ in Eq. (11) equal to zero; that is,

$$
\begin{gathered}
-2 i \omega A^{\prime}-2 i \omega \mu_{1} A-\mu_{2} g_{1}-3 \alpha_{3} A^{2} \bar{A}-\delta \omega^{2} A^{2} \bar{A}+3 \delta \omega^{2} A^{2} \bar{A} \\
-3 i G \omega^{3} A^{2} \bar{A}+\frac{1}{2} \eta_{1} f e^{i\left(\sigma T_{1}+\tau_{e}\right)}=0
\end{gathered}
$$

Note that satisfying Eq. (12) automatically satisfies its complex conjugate.

By substituting for $A$ and $\bar{A}$ from Eq. (9) into Eq. (12) obtains

$$
-i \omega\left(a^{\prime}+a i \beta^{\prime}\right)-i \omega \mu_{1} a-\frac{4}{3 \pi} i \mu_{2} \omega^{2} a^{2}+\left(\alpha_{e}-\frac{3}{8} i G \omega^{3}\right) a^{3}+\eta_{e} f e^{i \gamma}=0
$$

where

$$
\begin{aligned}
\alpha_{e} & =-\frac{3}{8} \alpha_{3}-\frac{1}{8} \delta \omega^{2}+\frac{3}{8} \delta \omega^{2} \\
\eta_{e} & =\frac{1}{2} \eta_{1} \\
\gamma & =\sigma T_{1}+\tau_{e}-\beta
\end{aligned}
$$

The parameters $\alpha_{e}$ and $\eta_{e}$ in Eqs (14) and (15) represent effective nonlinear restoring and forcing parameters, respectively. Separating Eq. (13) into imaginary and real parts and eliminating $\beta$ using Eq. (16) yields 


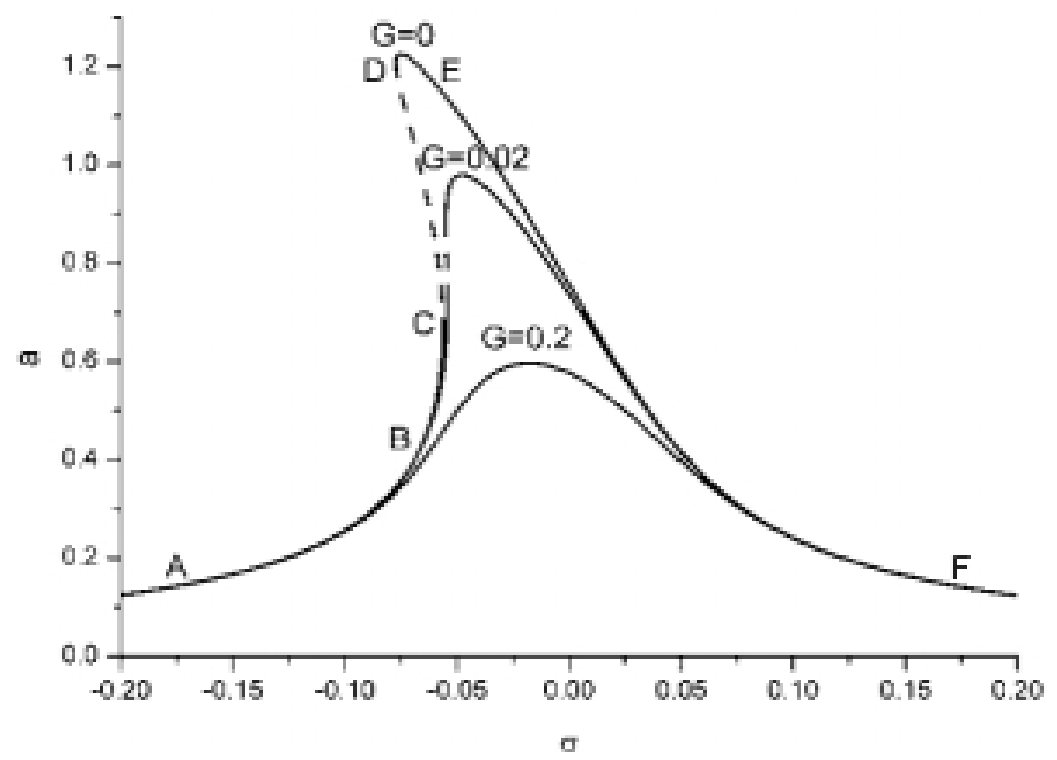

Fig. 1. Effect of varying the feedback gain on the frequency-response curves when $f=0.025$.

$$
\begin{aligned}
\omega a^{\prime} & =-\omega \mu_{1} a-\frac{4}{3 \pi} \mu_{2} \omega^{2} a^{2}-\frac{3}{8} G \omega^{3} a^{3}+\eta_{e} f \sin \gamma \\
\omega a \gamma^{\prime} & =a \omega \sigma+\alpha_{e} a^{3}+\eta_{e} f \cos \gamma
\end{aligned}
$$

where the prime represents differentiation with respect to the slow time scale $T_{1}$.

The performance of the control technique is evaluated by calculating the fixed points of Eqs (17) and (18) and examining their stability as a function of the parameters $f, \sigma$, and $G$. Setting $a^{\prime}=0$ and $\gamma^{\prime}=0$ yields

$$
\begin{aligned}
& \eta_{e} f \sin \gamma=\omega \mu_{1} a+\frac{4}{3 \pi} \mu_{2} \omega^{2} a^{2}+\frac{3}{8} G \omega^{3} a^{3} \\
& \eta_{e} f \cos \gamma=-a \omega \sigma-\alpha_{e} a^{3}
\end{aligned}
$$

The fixed points are given by the roots of

$$
\begin{aligned}
& \left(\omega \mu_{1} a+\frac{4}{3 \pi} \mu_{2} \omega^{2} a^{2}+\frac{3}{8} G \omega^{3} a^{3}\right)^{2}+\left(a \omega \sigma+\alpha_{e} a^{3}\right)^{2}-\left(\eta_{e} f\right)^{2}=0 \\
& \tan \gamma=\left(\omega \mu_{1}+\frac{4}{3 \pi} \mu_{2} \omega^{2} a+\frac{3}{8} G \omega^{3} a^{2}\right) /\left(-\omega \sigma-\alpha_{e} a^{2}\right)
\end{aligned}
$$

The form of Eqs (17) and (18) is not suitable for the stability analysis. Therefore, rewriting the $A$ in terms of Cartesian coordinate

$$
A=\frac{1}{2}\left[p\left(T_{1}\right)-i q\left(T_{1}\right)\right] e^{i\left(\nu T_{1}+\tau_{e}\right)}
$$

where $p$ and $q$ are real and $\nu=\sigma$. Substituting Eqs (23) into (12) and separating real and imaginary parts yields

$$
\begin{aligned}
p^{\prime}= & -\mu_{1} p-\nu q-\frac{\alpha_{e}}{\omega} p^{2} q-\frac{\alpha_{e}}{\omega} q^{3}-\frac{4}{3 \pi} \mu_{2} \omega \sqrt{p^{2}+q^{2}} p-\frac{3}{8} G \omega^{2} p^{3}-\frac{3}{8} G \omega^{2} p q^{2} \\
q^{\prime}= & -\mu_{1} q+\nu p+\frac{\alpha_{e}}{\omega} p^{3}+\frac{\alpha_{e}}{\omega} p q^{2}+\frac{\eta_{e} f}{\omega}-\frac{4}{3 \pi} \mu_{2} \omega \sqrt{p^{2}+q^{2}} q \\
& -\frac{3}{8} G \omega^{2} p^{2} q-\frac{3}{8} G \omega^{2} q^{3}
\end{aligned}
$$




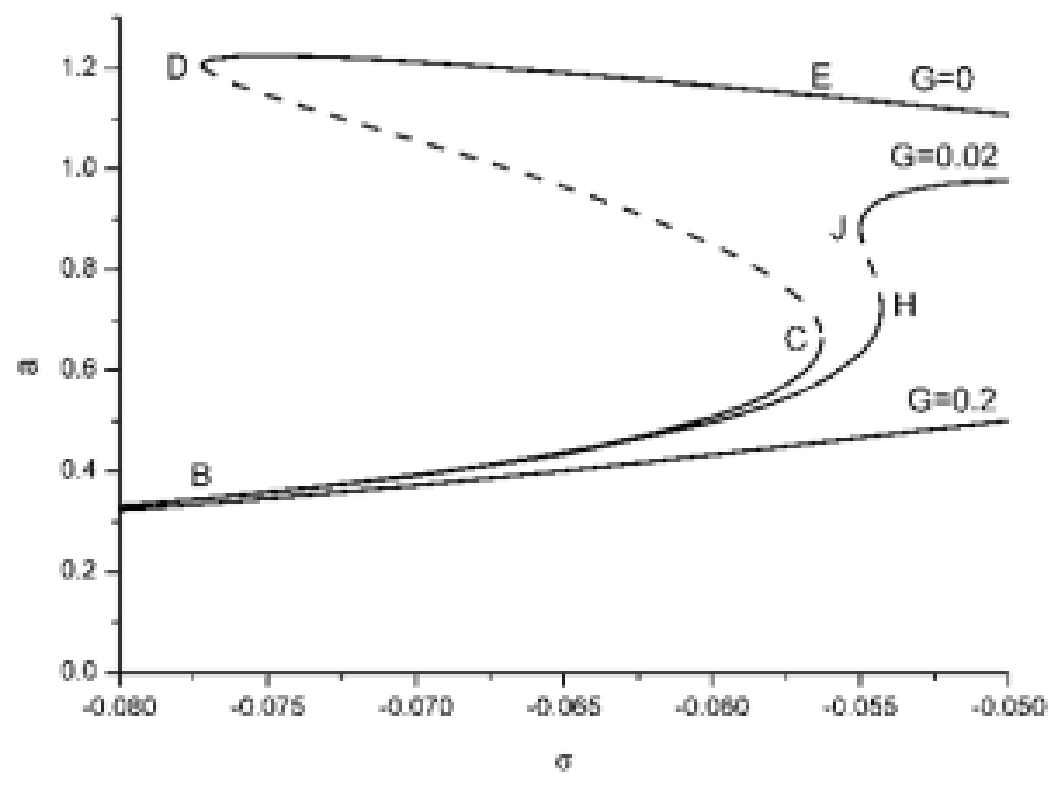

Fig. 2. An enlargement of the frequency response curves near the saddle-node bifurcation point $C$.

The performance of the control is evaluated by calculating the equilibrium solutions of Eqs (24) and (25) and examining their stability as a function of the parameters $f, \sigma$, and $G$. Setting the time derivatives in Eqs (24) and (25) equal to zero and solve the resulting system of algebraic equations for $p$ and $q$ for a specified value of either $\sigma$, which is a measure of the detuning of the primary resonance, or $f$, which is a measure of the forcing amplitude. The amplitude $a$ of the response of the plant is then calculated from $a=\sqrt{p^{2}+q^{2}}$.

The classical method of stability analysis in which the behavior of the nonlinear system in the neighborhood of the equilibrium state is approximated by the behavior of the associated linear system is adopted in this paper. Although the Lyapunov's direct method is not restricted to an infinitesimal neighborhood of the equilibrium state, the principal limitations of the Lyapunov's direct method are the lack of general methods for the construction of Lyapunov functions and the lack of uniqueness. The stability of a particular equilibrium solution is determined by examining the eigenvalues of the Jacobian matrix of the right-hand sides of Eqs (24) and (25). The eigenvalues of the Jacobian matrix provide information about the local stability of the equilibrium solution. If the real part of each eigenvalue of the Jacobian matrix is negative, the corresponding equilibrium solution is asymptotically stable. If the real part of any of the eigenvalues is positive, the corresponding equilibrium solution is unstable. According to references $[18,19]$, the stability of the equilibrium solution is determined by the roots of

$$
\left|\begin{array}{cc}
J_{11}-\lambda J_{12} \\
J_{21} & J_{22}-\lambda
\end{array}\right|=0
$$

where

$$
\begin{aligned}
& J_{11}=-\mu_{1}-\frac{2 p q \alpha_{e}}{\omega}-\frac{4 p^{2} \mu_{2} \omega}{3 \pi \sqrt{p^{2}+q^{2}}}-\frac{4 \sqrt{p^{2}+q^{2}} \mu_{2} \omega}{3 \pi}-\frac{9}{8} G p^{2} \omega^{2}-\frac{3}{8} G q^{2} \omega^{2} \\
& J_{12}=-\nu-\frac{p^{2} \alpha_{e}}{\omega}-\frac{3 q^{2} \alpha_{e}}{\omega}-\frac{4 p q \mu_{2} \omega}{3 \pi \sqrt{p^{2}+q^{2}}}-\frac{3}{4} G p q \omega^{2} \\
& J_{21}=\nu+\frac{3 p^{2} \alpha_{e}}{\omega}+\frac{q^{2} \alpha_{e}}{\omega}-\frac{4 p q \mu_{2} \omega}{3 \pi \sqrt{p^{2}+q^{2}}}-\frac{3}{4} G p q \omega^{2} \\
& J_{22}=-\mu_{1}+\frac{2 p q \alpha_{e}}{\omega}-\frac{4 q^{2} \mu_{2} \omega}{3 \pi \sqrt{p^{2}+q^{2}}}-\frac{4 \sqrt{p^{2}+q^{2}} \mu_{2} \omega}{3 \pi}-\frac{3}{8} G p^{2} \omega^{2}-\frac{9}{8} G q^{2} \omega^{2}
\end{aligned}
$$




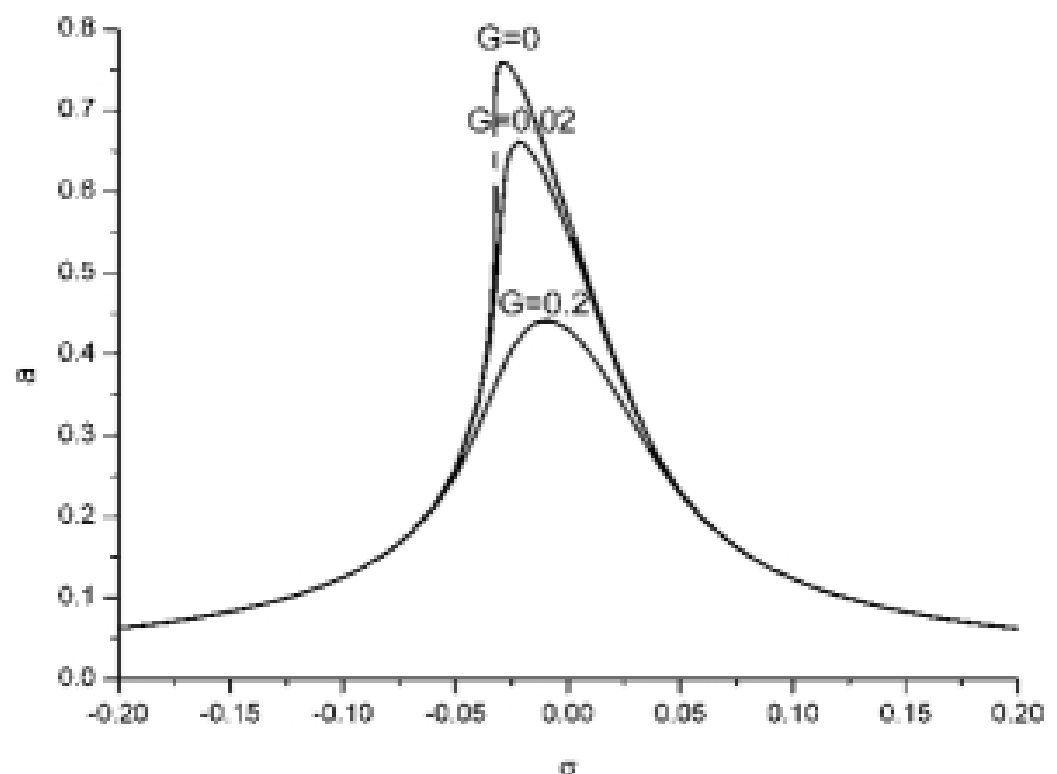

Fig. 3. Effect of varying the feedback gain on the frequency-response curves when $f=0.0125$.

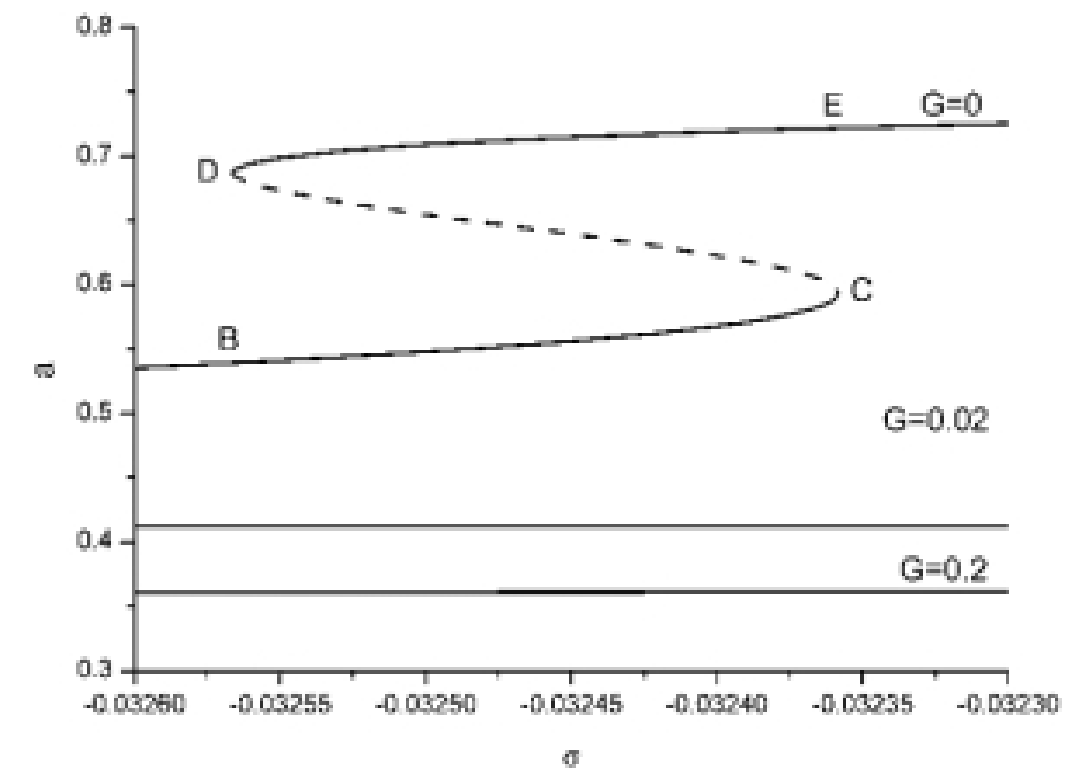

Fig. 4. An enlargement of the frequency response curves near the saddle-node bifurcation point $C$.

Due to the complexity in obtaining a closed form solution to Eq. (26), numerical methods are resorted to investigate the stability of the equilibrium solutions.

\section{Frequency-response curves}

The various parameters of the nonlinear plant used in the paper are $, \mu_{2}=0.02, \alpha_{e}=0.05, \omega=1, \eta_{e}=1$. The typical frequency-response curves $(f=0.025)$ of the open- and closed-loop cases of the nonlinear plant are shown 


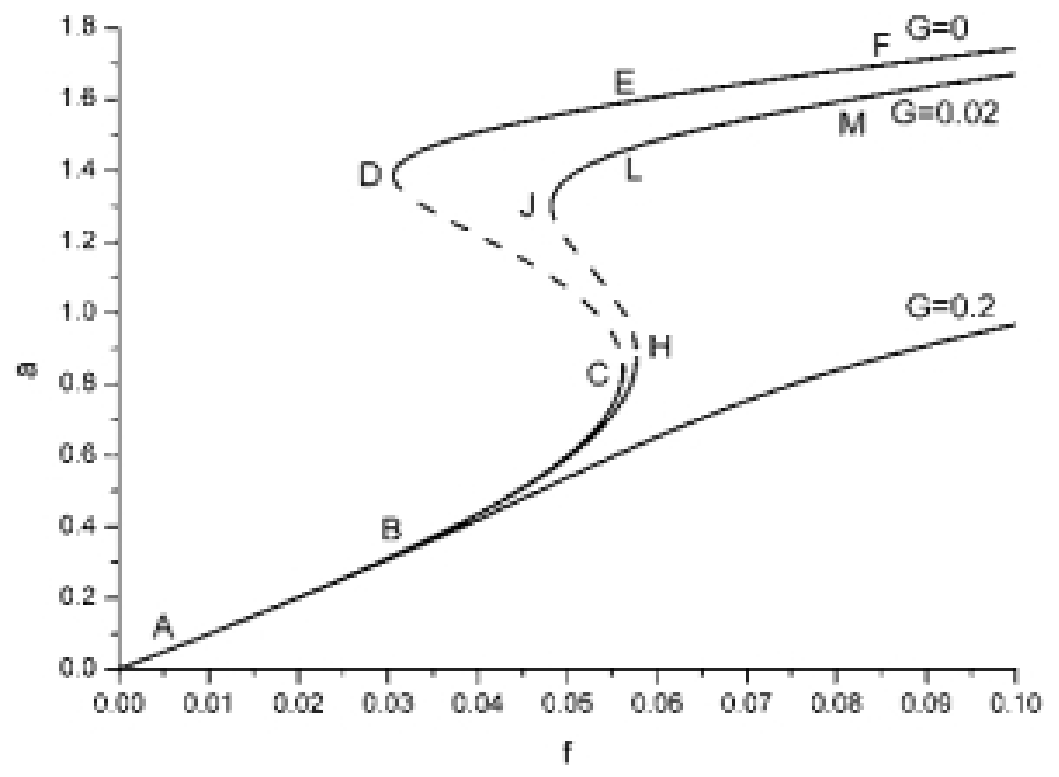

Fig. 5. Effect of varying the feedback gain on the force-response curves when $\sigma=-0.1$.

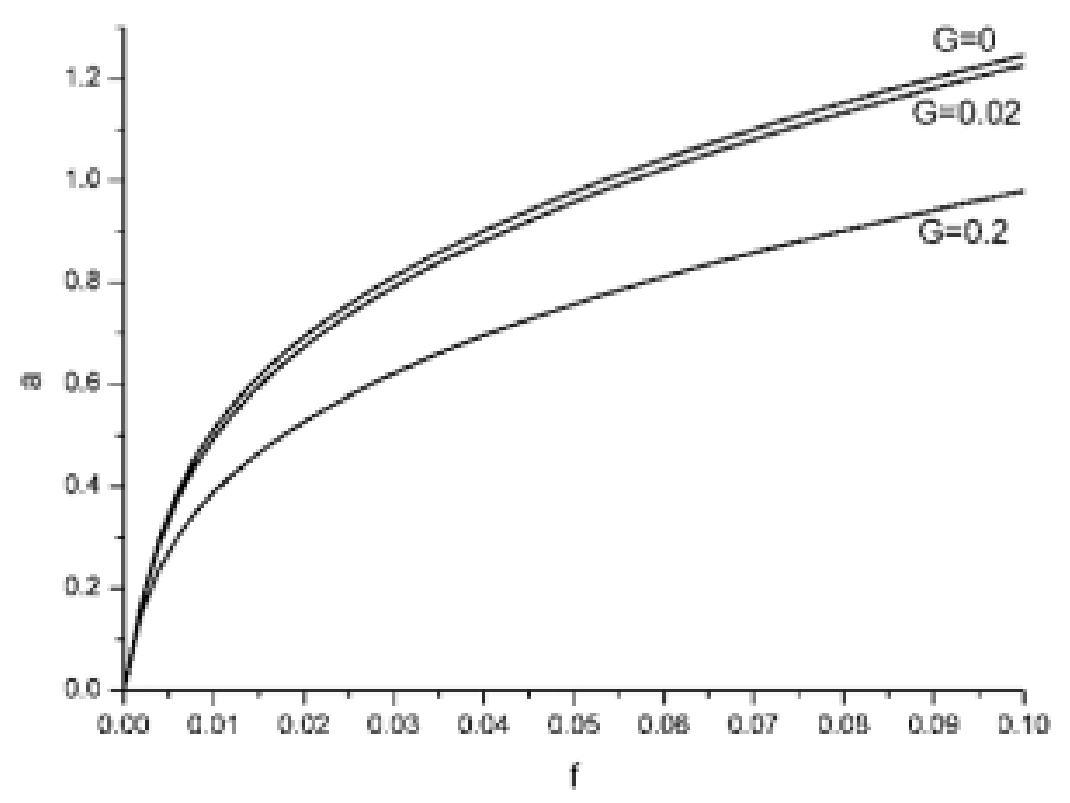

Fig. 6. Effect of varying the feedback gain on the force-response curves when $\sigma=0$.

in Fig. 1, and an enlargement of the frequency-response curves is shown in Fig. 2. The response amplitudes depend on the value of $\sigma$ and the plant's initial conditions. The solid lines correspond to stable solutions, while the dashed lines correspond to unstable solutions.

The open-loop response (i.e., $G=0$ ) is considered first. As $\sigma$ is increased from point $A$, the response amplitude increases along the curve $A C$. When $\sigma$ reaches $\sigma_{C}$, the equilibrium solution undergoes a saddle-node bifurcation, and the response amplitude jumps up to the high-amplitude response in point $E$. A further increase in $\sigma$ leads to the response amplitude decreasing along the curve $E F$. On the other hand, when $\sigma$ is decreased from point $F$, the response amplitude increases along the curve $F D$. When $\sigma$ reaches $\sigma_{D}$, the response undergoes a saddle-node 


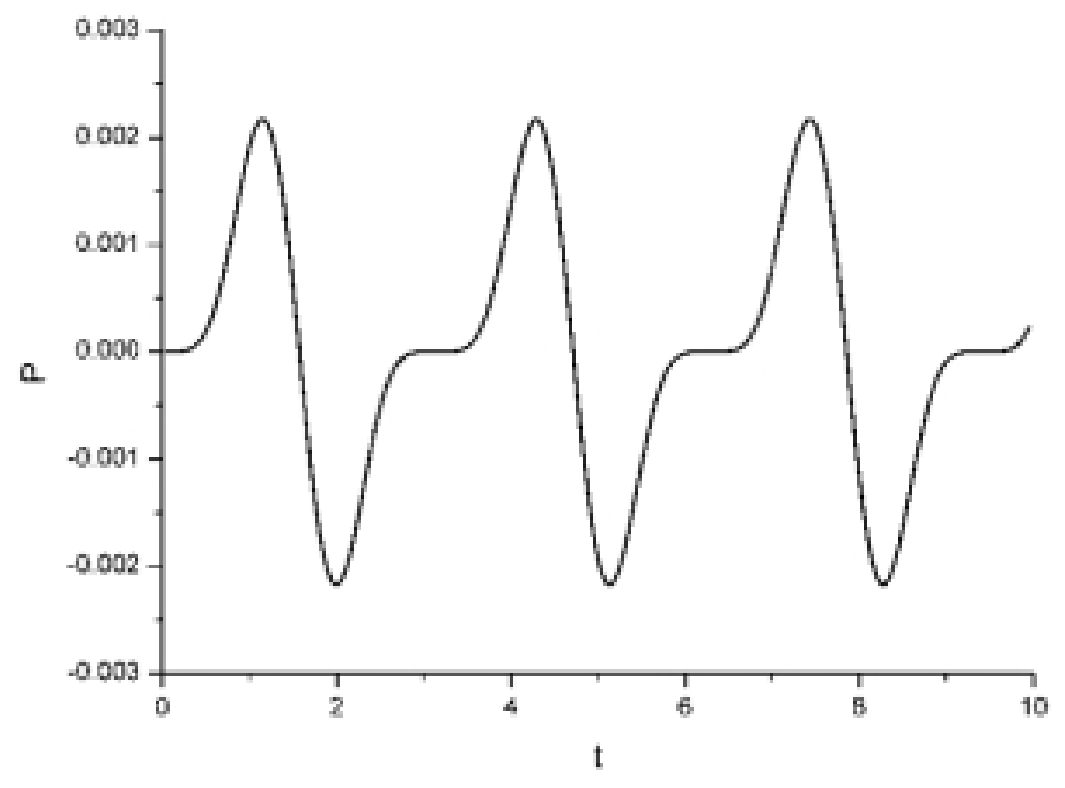

Fig. 7. Time traces of the instantaneous power when $f=0.025, \sigma=0$ and $K=0.5$.

bifurcation, and the response amplitude jumps down to the low-amplitude response in point $B$. A further decrease in $\sigma$ leads to the response amplitude decreasing along the curve $B A$.

Next, the response of the closed-loop system (i.e., $G \neq 0$ ) is analyzed. Two different feedback gains, i.e., $G=0.02$ and $G=0.2$, are considered. In the first case, the response undergoes saddle-node bifurcations similar to those of the open-loop case. However, the response amplitude is decreased, and the positions of the saddle-node bifurcations are shifted from point $C$ to point $H$ and from point $D$ to point $J$ (Fig. 2). In the second case, superior result is achieved. The saddle-node bifurcations and the jump phenomenon are eliminated, and the response is suppressed further. From Figs 1 and 2, it is clear that, as the feedback gain is increased, the response amplitude of the nonlinear plant is decreased. Figure 1 also indicates that the control law leads to effective vibration suppression and bifurcation control in a wide range of frequency around resonance when $G=0.2$.

The frequency-response curves $(f=0.0125)$ of the open- and closed-loop cases of the nonlinear plant are shown in Fig. 3, and an enlargement of the frequency-response curves is shown in Fig. 4. It can be seen from Figs 1 and 3 that, as the amplitude of excitation is increased, the frequency-response curve bends away from the linear curve, resulting in larger multivalued region. The multivaluedness is responsible for jumps. It is also noted here that, as the forcing amplitude is increased, the nonlinearity tends to dominate the response.

\section{Force-response curves}

The typical open- and closed-loop force-response curves $(\sigma=-0.1)$ are shown in Fig. 5. Again, the open-loop response is considered first. As $f$ is increased from point $A$, the response amplitude increases along the curve $A C$. When $f$ reaches $f_{C}$, the equilibrium solution undergoes a saddle-node bifurcation, and the response amplitude jumps up to the high-amplitude response in point $E$. A further increase in $f$ leads to a higher response amplitude, the response amplitude traces the curve $E F$. On the other hand, when $f$ is decreased from point $F$, the response amplitude decreases along the curve $F D$. When $f$ reaches $f_{D}$, the response undergoes a saddle-node bifurcation, and the response amplitude jumps down to the low-amplitude response in point $B$. A further decrease in $f$ leads to the response amplitude decreasing along the curve $B A$.

The response of the closed-loop system is considered next. Again, the force-response for two different values of the feedback gain is analyzed. When $G=0.02$, the force-response curve is similar to the uncontrolled forceresponse curve. The response undergoes identical saddle-node bifurcation, however, the locations of the saddle-node 


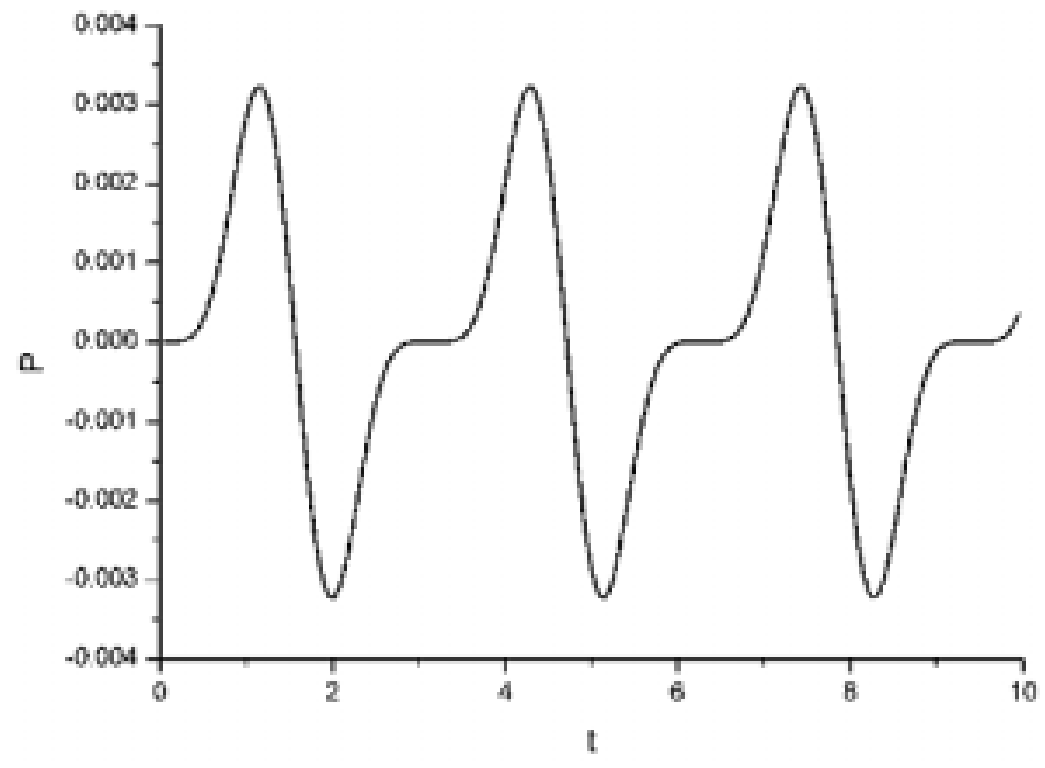

Fig. 8. Time traces of the instantaneous power when $f=0.025, \sigma=0$ and $K=0.1$.

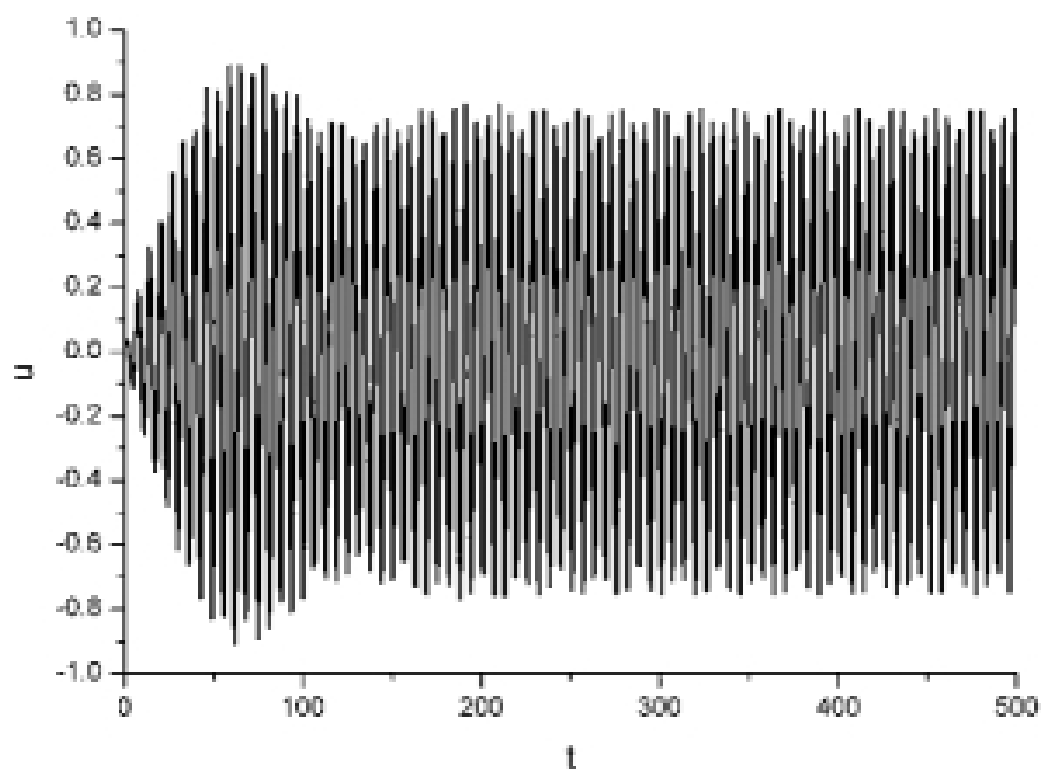

Fig. 9. Numerical simulation of the time response for the open-loop case when $\Omega=1.0, u(0)=0.02$ and $\dot{u}(0)=0$.

bifurcation points are shifted from points $C$ and $D$ to points $H$ and $J$, respectively, resulting in a smaller hysteretic region. Furthermore, the response amplitude is reduced from the curve $D E F$ to the curve $J L M$. As the feedback gain is further increased, the saddle-node bifurcations at points $C$ and $D$ are eliminated, and the jump-up and jump-down due to the saddle-node bifurcations are thus eliminated. Additionally, the amplitude of the response is further reduced.

The open- and closed-loop force-response curves $(\sigma=0)$ are shown in Fig. 6. It is clear from Figs 5 and 6 that, as the excitation amplitude is increased, the response amplitude of the plant is increased. 


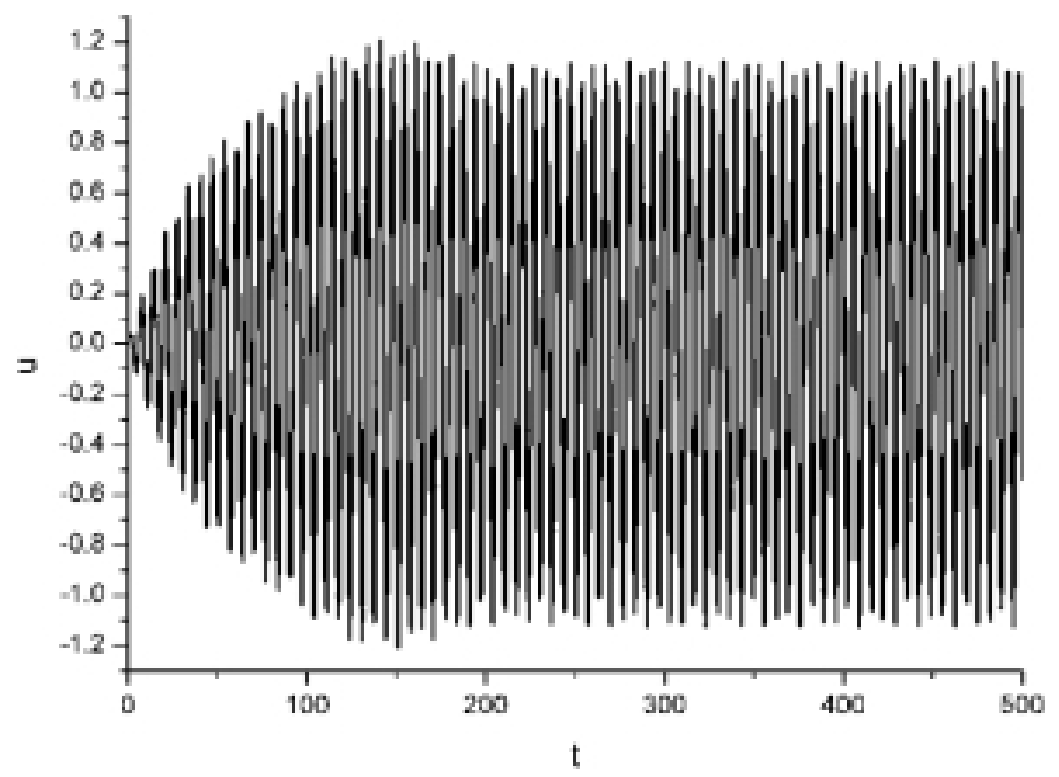

Fig. 10. Numerical simulation of the time response for the open-loop case when $\Omega=0.95, u(0)=0.02$ and $\dot{u}(0)=0$.

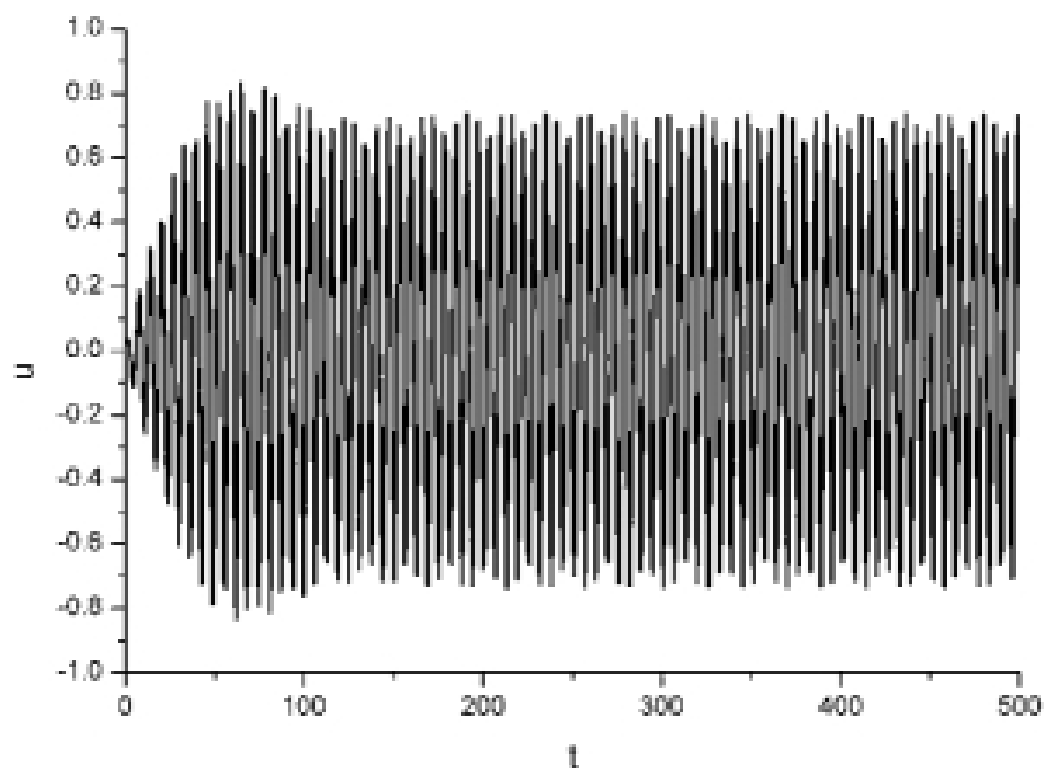

Fig. 11. Numerical simulation of the time response when $\Omega=1.0, G=0.02, u(0)=0.02$ and $\dot{u}(0)=0$.

\section{Power requirement}

One performance metric that is considered as a criterion to select a control strategy is the power requirement. To this end, the instantaneous power for the cubic velocity feedback control is computed. The modulation equations for the amplitude and phase are already obtained in Section 2, the response amplitude $a$ is determined from the roots of Eq. (21).

Only perfect resonance (i.e., $\sigma=0$ ) is considered in this section. Assuming $a_{o l}$ is the open-loop response amplitude and $a_{c l}$ is the close-loop response amplitude. Given the required reduction in the response amplitude, i.e., $a_{c l}=K a_{o l}$, where $K<1$, then the feedback gain $G$ can be determined. 


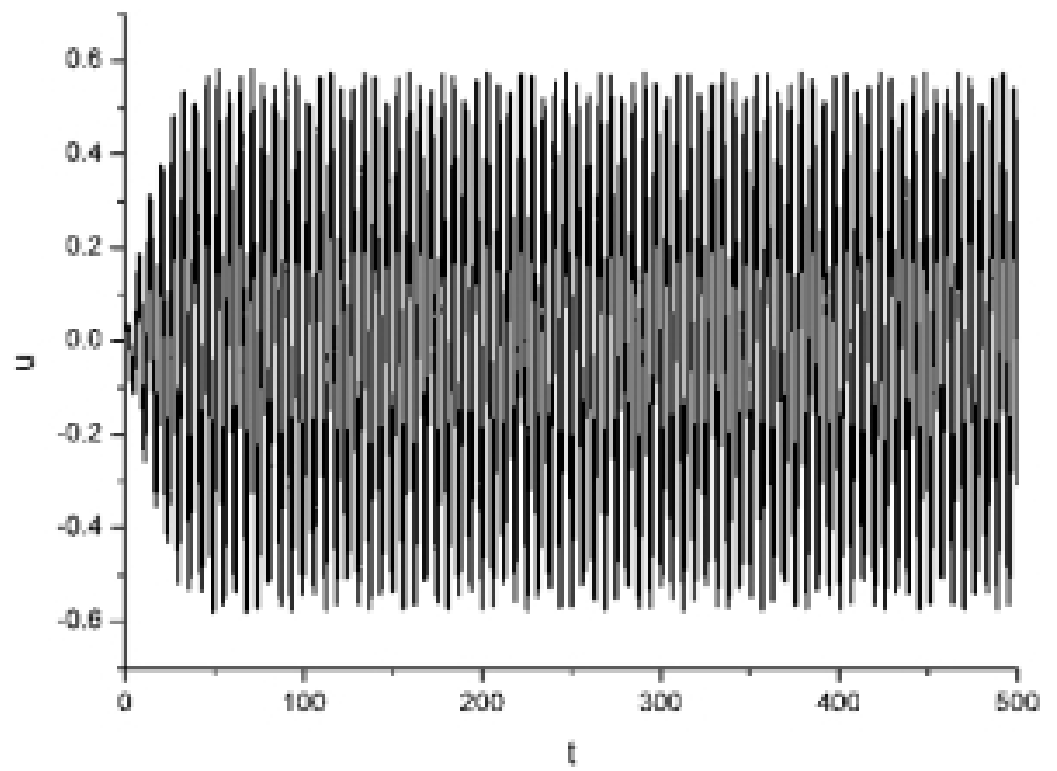

Fig. 12. Numerical simulation of the time response when $\Omega=1.0, G=0.2, u(0)=0.02$ and $\dot{u}(0)=0$.

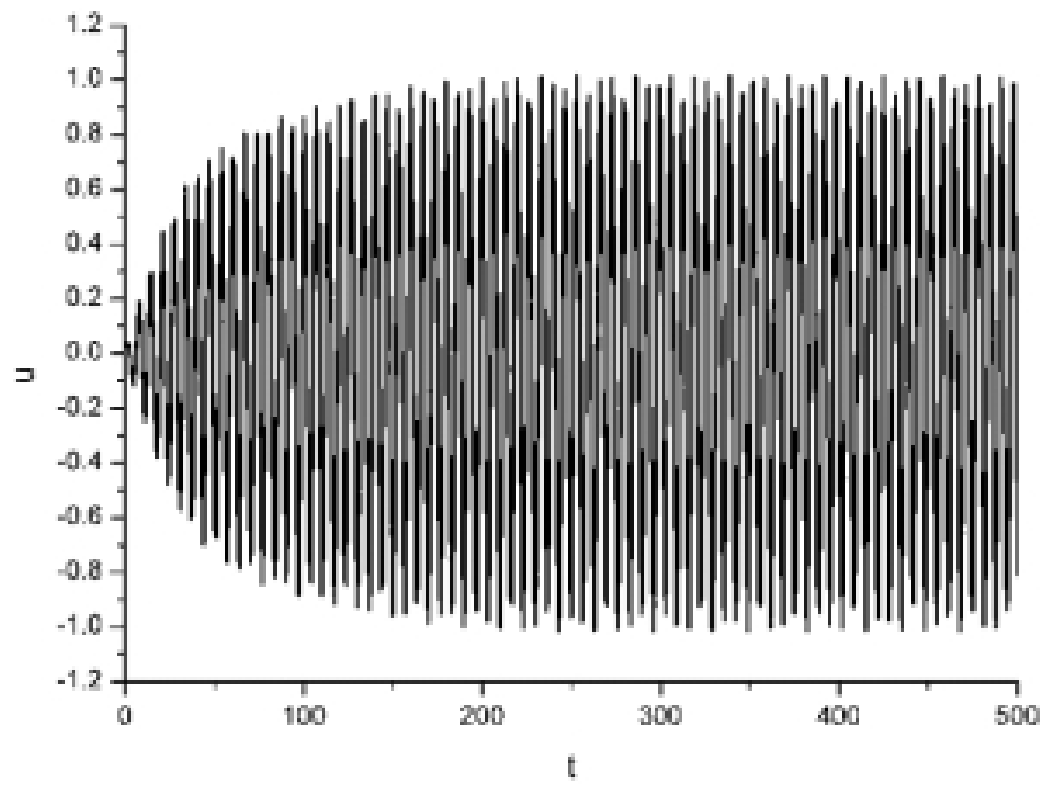

Fig. 13. Numerical simulation of the time response when $\Omega=0.95, G=0.02, u(0)=0.02$ and $\dot{u}(0)=0$.

Assuming the response to be a single tone, one can find that $u=a \cos t$. If the actuator is made of piezoceramics, then the voltage signal is given by

$$
V=G \dot{u}^{3}
$$

The instantaneous power is defined by

$$
P=V I
$$

Here, $V$ is the control voltage, and the resulting current $I$ is given by 


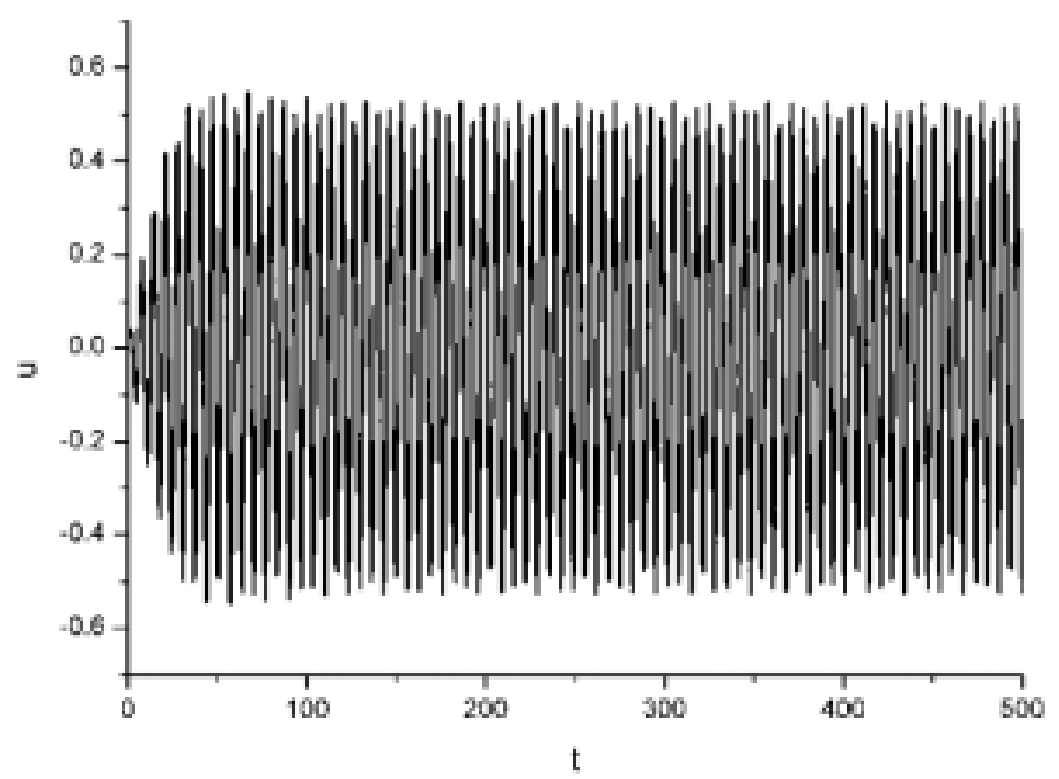

Fig. 14. Numerical simulation of the time response when $\Omega=0.95, G=0.2, u(0)=0.02$ and $\dot{u}(0)=0$.

$$
I=C \frac{d V}{d t}
$$

where $C$ is the effective capacitance of the actuator. Substituting Eqs (31) and (33) into Eq. (32) obtains, after trigonometric manipulations,

$$
P=\frac{3 C G^{2} \omega^{7} a^{6}}{32}(5 \sin 2 \omega t-4 \sin 4 \omega t+\sin 6 \omega t)
$$

where $P$ is the power required to suppress the vibration of the plant under the nonlinear cubic velocity feedback control law. Figures 7 and 8 show the instantaneous powers calculated from Eq. (34).

\section{Numerical simulations}

To validate the results of perturbation analysis, the following differential equation is integrated numerically using a non-stiff implicit Adams integrator scheme.

$$
\begin{aligned}
& \frac{d^{2} u}{d t^{2}}+\omega^{2} u+2 \mu_{1} \frac{d u}{d t}+\mu_{2} \frac{d u}{d t}\left|\frac{d u}{d t}\right|+\alpha_{3} u^{3}+\delta\left(\frac{d u}{d t}\right)^{2} u+\delta u^{2} \frac{d^{2} u}{d t^{2}} \\
& =\eta_{1} f \cos \left(\Omega t+\tau_{e}\right)-G \dot{u}^{3}
\end{aligned}
$$

where $\mu_{1}=0.01, \mu_{2}=0.02, \alpha_{3}=-0.08, \delta=0.08, \eta_{1}=2, \omega=1, \tau_{e}=0$ and $f=0.025$. The numerical simulations are performed for different values of the forcing frequency $\Omega$ and the feedback gain $G$. The time responses for the open-loop case (i.e., $G=0$ ) when $\Omega=1.0$ (i.e., $\sigma=0$ ) and $\Omega=0.95$ (i.e., $\sigma=-0.05$ ) are shown in Figs 9 and 10. The time responses when $\Omega=1.0$ for $G=0.02$ and $G=0.2$ are shown in Figures 11 and 12. The time responses when $\Omega=0.95$ for $G=0.02$ and $G=0.2$ are shown in Figs 13 and 14. Comparing Fig. 1 with Figs 9-14, it can be seen that the response amplitudes predicted by the perturbation solutions are in good agreement with the results of the numerical simulations. The results of numerical simulations shown in Figs 9-14 also indicate that large-amplitude of the plant can be reduced by introducing the nonlinear cubic velocity feedback control strategy. 


\section{Conclusions}

The use of cubic velocity feedback control strategy to suppress the high-amplitude vibration of a nonlinear plant to a primary resonance excitation is investigated. Two first-order differential equations governing the amplitude and phase of the response are obtained using the method of multiple scales. The stability analysis for the open- and closed-loop responses of the nonlinear system is conducted and the parametric investigation of the performance of the control law is carried out. It is shown that cubic velocity feedback control technique is successful in reducing the response amplitude of the nonlinear plant as well as eliminating the multiple responses. Also, the response amplitude is decreased as the feedback gain is increased. To validate the perturbation solutions, the numerical simulations of the nonlinear plant are performed. Good agreement between the perturbation results and numerical simulations is found. The work presented in this paper can be expanded and enhanced by conducting the experiments to validate the cubic velocity feedback control model, implementing the other control strategies and comparing with that studied here, and performing the study of the robustness of the cubic velocity feedback control technique for the multi-degree-of-freedom systems.

\section{Acknowledgement}

The authors would like to thank the reviewers for their helpful and important comments and suggestions.

\section{References}

[1] A.M. Abou-Rayan, A.H. Nayfeh, D.T. Mook and M.A. Nayfeh, Nonlinear response of a parametrically excited buckled beam, Nonlinear Dynamics 4(1993), 499-525.

[2] A.A. Afaneh and R.A. Ibrahim, Nonlinear response of an initially buckled beam with 1:1 internal resonance to sinusoidal excitation, Nonlinear Dynamics 4(1993), 547-571.

[3] T.T. Soong, State-of-the-art review: active structural control in civil engineering, Engineering Structures 10(1988), 74-84.

[4] J. Suhardjo, B.F. Spencer. Jr and M.K. Sain, Non-linear optimal control of a Duffing system, International Journal of Nonlinear Mechanics 27(1992), 157-172.

[5] M. Abdel-Rohman and A.H. Nayfeh, Active control of nonlinear oscillations in bridges, Journal of Engineering Mechanics 113(1987), $335-348$.

[6] J.N. Yang, F.X. Long and D. Wong, Optimal control of nonlinear structures, Transactions of ASME, Journal of Applied Mechanics 55 (1988), 931-938

[7] S.F. Masri, R.K. Miller, T.J. Dehghanyar and T.K. Caughey, Active parameter control of nonlinear vibrating structures, Transactions of ASME, Journal of Applied Mechanics 56 (1989), 658-666.

[8] J.L. Moiola, H.G. Chiacchiarini and A.C. Desages, Bifurcations and Hopf degeneracies in nonlinear feedback systems with time delay, International Journal of Bifurcation and Chaos 6 (1996), 661-672.

[9] H. Hu, E.H. Dowell and L.N. Virgin, Resonances of a harmonically forced Duffing oscillator with time delay state feedback, Nonlinear Dynamics 15 (1998), 311-327.

[10] A.A. El-Badawy and A.H. Nayfeh, Control of a directly excited structural dynamic model of an F-15 tail section, Journal of the Franklin Institute 338 (2001), 133-147.

[11] O.C. Pinto and P.B. Goncalves, Active non-linear control of buckling and vibrations of a flexible buckled beam, Chaos, Solitons and Fractals 14 (2002), 227-239.

[12] A. Maccari, The response of a parametrically excited van der Pol oscillator to a time delay state feedback, Nonlinear Dynamics 26 (2001), $105-119$.

[13] A. Maccari, Vibration control for the primary resonance of the van der Pol oscillator by a time delay state feedback, International Journal of Nonlinear Mechanics 38 (2003), 123-131.

[14] H. Yabuno, S. Saigusa and N. Aoshima, Stabilization of the parametric resonance of a cantilever beam by bifurcation control with a piezoelectric actuator, Nonlinear Dynamics 26 (2001), 143-161.

[15] S.S. Oueini and A.H. Nayfeh. Single-mode control of a cantilever beam under principal parametric excitation, Journal of Sound and Vibration 224 (1999), 33-347.

[16] L. Chen, T. Cao, F. He and K. Sammut, Bifurcation control of a flexible beam under principal parametric excitation, in: Proceedings of the American Control Conference, Chicago, Illinois, 2000.

[17] A.H. Nayfeh and T.D. Mook, Nonlinear oscillations, Wiley, New York 1979

[18] A.H. Nayfeh and B. Balachandran, Applied Nonlinear Dynamics, Wiley, New York, 1995.

[19] R. Seydel, Practical Bifurcation and Stability Analysis: from Equilibrium to Chaos, Springer-Verlag, New York, 1994. 

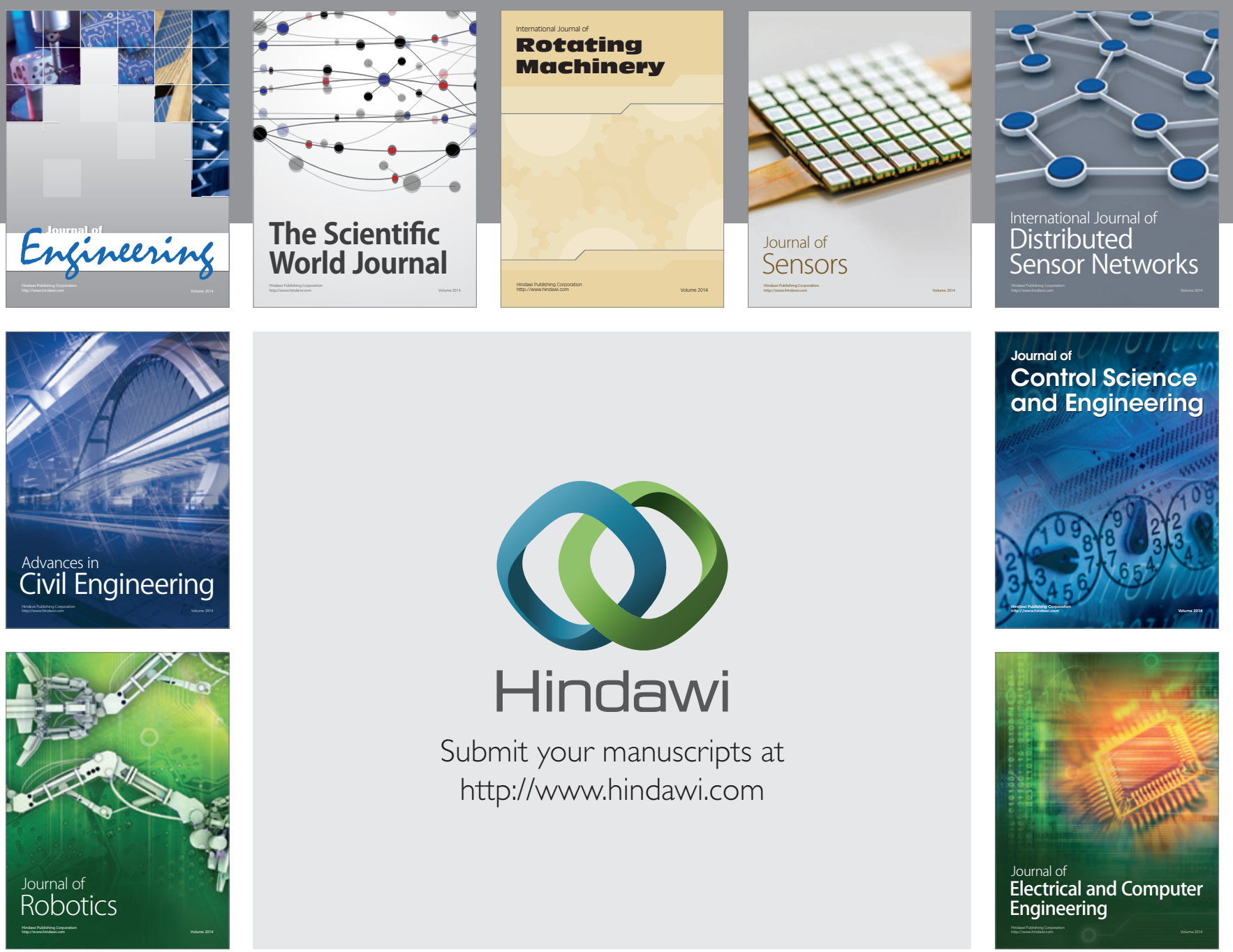

Submit your manuscripts at

http://www.hindawi.com
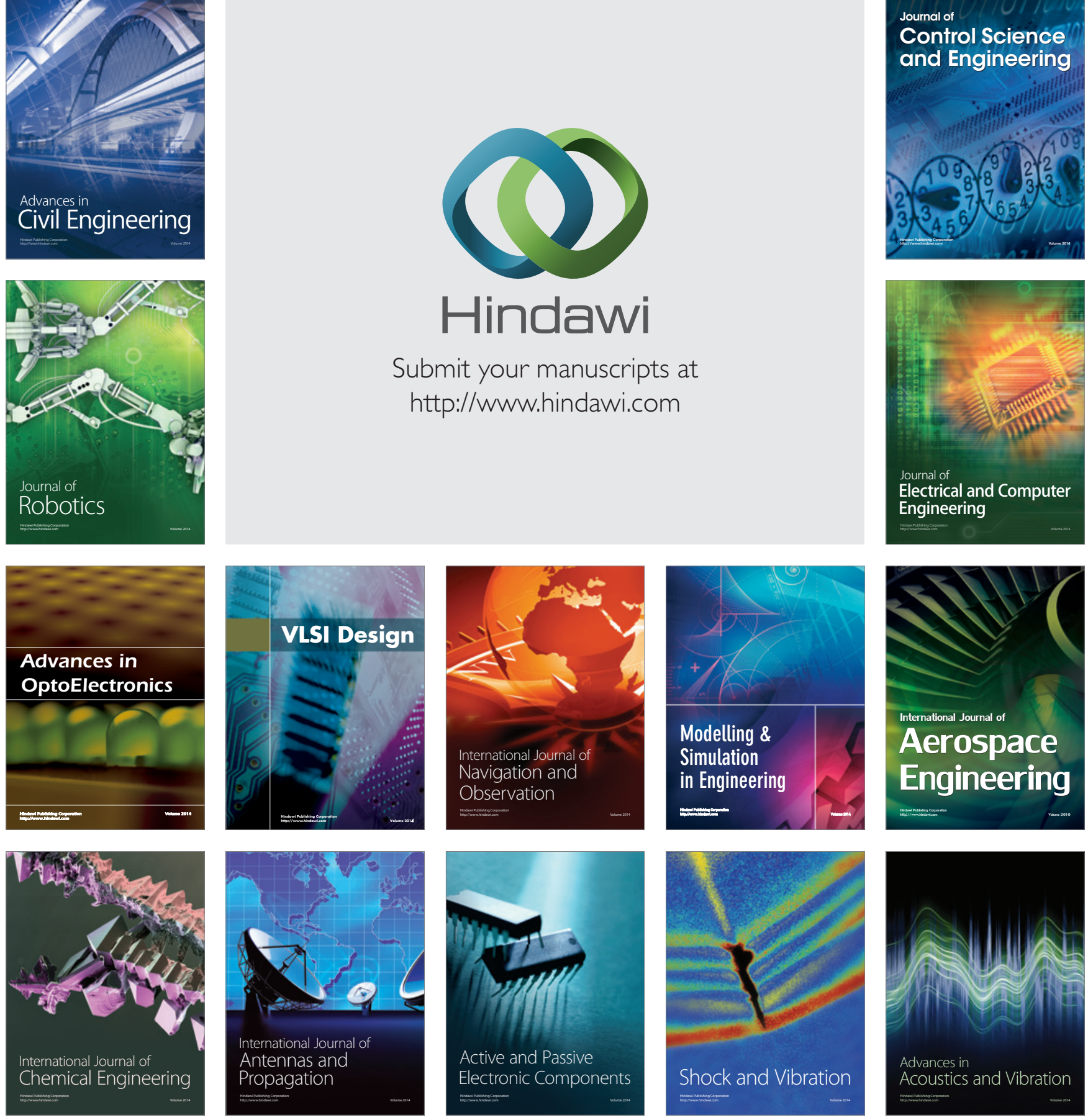